DOI: https://doi.org/10.11144/Javeriana.upsy16-4.rrea

\title{
Resiliencia y rendimiento escolar en adolescentes de Lima y de Santiago de Chile de sectores vulnerables*
}

\section{Resilience and Academic Performance of Lima and Santiago de Chile Adolescents from Vulnerable Social Level}

\author{
Marco Antonio Villalta Paucar ${ }^{a}$ \\ Universidad de Santiago de Chile, Chile \\ ORCID: http://orcid.org/0000-0002-7553-925X \\ Ana Esther Delgado Vásquez \\ Universidad Nacional Mayor de San Marcos, Perú \\ Luis Miguel Escurra Mayaute \\ Universidad Nacional Mayor de San Marcos, Perú \\ William Torres ACUÑa \\ Universidad Ricardo Palma, Perú
}

\footnotetext{
a Autor de correspondencia. Correo electrónico: marco.villalte@usach.cl

Para citar este artículo: Villalta, M., Delgado, A., Escurra, L., \& Torres, W. (2017). Resiliencia y Rendimiento Escolar en Adolescentes de Lima y de Santiago de Chile de Sectores Vulnerables. Universitas Psychologica, 16(4), 1-10. https://doi.org/10.11144/Jav eriana.upsy16-4.rrea
}

\section{RESUMEN}

El objetivo del estudio es proponer instrumentos de medición de la resiliencia relacionada al rendimiento escolar, válida para adolescentes y jóvenes de sectores sociales vulnerables de zonas urbanas de dos países latinoamericanos. El método de investigación está basado en el análisis del comportamiento estadístico de los ítems y dimensiones de la resiliencia de la escala SV-RES en 764 adolescentes de zonas urbanas vulnerables de Lima-Perú y 1145 de Santiago de Chile, con alto y bajo rendimiento escolar de sectores sociales de alta vulnerabilidad social. Se realizó Análisis Factorial Exploratorio (AFE) y el análisis de validez convergente y discriminante. Los resultados sugieren nuevas dimensiones de la resiliencia vinculada al rendimiento académico, diferenciadas para adolescentes de Lima y de Santiago de Chile.

Palabras clave

resiliencia; medición; análisis factorial exploratorio; educación.

\footnotetext{
ABSTRACT

The aim of the study is to propose measuring instruments resilience related to school performance valid for adolescents from vulnerable social groups in urban areas of two South American countries. The research method is based on analysis of the statistical results of the items and dimensions of the resilience of the SV-RES Scale in 764 adolescents from vulnerable urban areas of Lima, Peru and 1,145 from Santiago de Chile with high and low academic performance of sectors with social vulnerability. Exploratory Factor Analysis (AFE), convergent and discriminant validity analysis was performed. The results suggest new different dimensions of resilience linked to academic performance on adolescents of Lima and Santiago de Chile.

Keywords

resilience; measurement; exploratory factor analysis; education.
} 
Existe diversidad de aproximaciones conceptuales y operativas al constructo resiliencia, algunos casos lo describen como rasgo individual y otros como proceso dinámico (Luthar, Cicchetti, \& Becker, 2000); algunas tradiciones de estudio focalizan la resiliencia como resistencia al estrés y otros como mecanismo de superación (Brandão, Mahfoud, \& Gianordoli-Nascimento, 2011); otras ponen de relieve la resiliencia como modo de intervención que modifique prácticas sociales (Llobet \& Wegsman, 2004). Esta diversidad conceptual confluye en personas -individuos o colectivosque viven situación de riesgo o adversidad, lo cual sustenta el desarrollo de propuestas de medición de la resiliencia en sus resultados comportamentales y de respuesta fisiológica (Cortés \& Cruz, 2011; Ospina, 2007).

La posibilidad de medición de la resiliencia se sustenta en la especificidad de la población que delimita el constructo para desarrollar nuevas tendencias de investigación (Grotberg, 2005) y el conocimiento acumulado sobre las relaciones probabilísticas entre adversidad, factores de riesgo y los resultados en la salud de las personas (Fergus \& Zimmerman, 2005; Morelato, 2014).

Se reportan en revistas científicas indexadas, propuestas específicas de medición de la resiliencia para el contexto latinoamericano, como la Escala RESI-M empleada en población joven (Lever \& Valdez, 2010), y el Inventario IRES validado con madres (Gaxiola, Frías, Hurtado, Salcido, \& Figueroa, 2011), ambos en México; el cuestionario CRE-U diseñado para población universitaria de Colombia (Peralta, Ramírez, \& Castaño, 2006); el Inventario de Factores Personales de la Resiliencia, propuesto para población peruana (Salgado, 2005); y la Escala SV-RES propuesta para jóvenes y adultos de población chilena (Saavedra \& Villalta, 2008a; Villalta \& Delgado, 2013). Las propuestas de medición coinciden en señalar factores o dimensiones que operan de modo aditivo o específico para explicar el fenómeno resiliencia.

Entre las propuestas latinoamericanas de medición de la resiliencia, la escala SV-RES de Saavedra y Villalta (2008a) es la que considera mayor cantidad de factores o dimensiones de la resiliencia: 12 factores sustentados en validez curricular, cuya versión original considera el mayor número de ítems: 60 reactivos en la versión original, en comparación con sus pares latinoamericanaos. La escala SV-RES ha sido utilizada en diversos tipos de poblaciones en situaciones de riesgo o adversidad, y en otros países latinoamericanos.

El objetivo del presente artículo es proponer una versión de medición de la resiliencia a la exclusión social, que sea estadísticamente válida para adolescentes y jóvenes de sectores sociales vulnerables de zonas urbanas de dos países latinoamericanos. Para lograr esto, se analizan las propiedades psicométricas de la escala SVRES en población adolescente y joven de sectores sociales vulnerables de Perú y Chile, y como indicador de logro inclusión se considera el rendimiento escolar.

\section{Resiliencia y educación: algunos antecedentes de su medición}

Específicamente en el campo educativo, los estudios en resiliencia se pueden agrupar de modo tentativo y no totalmente excluyente unos de otros - entre aquellos que abordan la resistencia a situaciones que afectan la salud mental y bienestar (Gibbs \& Miller, 2014; Gloria, Faulk, \& Steinhardt, 2013; Moreno \& Saiz, 2014), y aquellos estudios que indagan la relación entre situaciones de adversidad y el rendimiento académico (Gaxiola, González, \& Gaxiola, 2013; Kotzé \& Kleynhans, 2013).

Gaxiola et al. (2011) indican que la medición de la resiliencia requiere de tres elementos: (1) identificar el factor de riesgo o situación de adversidad; (2) la definición de características disposicionales personales y (3) el logro o meta que es comúnmente afectada por la adversidad, pero favorecida por el proceso resiliente. Los elementos 1 y 3 son datos que ofrece la realidad. El elemento 2 requiere de una elaboración teórica que sustente factores o dimensiones personales y contextuales, que inciden en el logro de resultados positivos. Una de las propuestas es 
la Escala SV-RES en la que se profundiza a continuación.

En la escala SV-RES (Saavedra \& Villalta, 2008a) se define la resiliencia como capacidad humana, constituida de modo interactivo en la historia del sujeto, para generar acciones que posibilitan el desarrollo positivo frente a la adversidad (Villalta, Saavedra \& Escurra, 2007). Esta vincula los estudios de Edith Grotberg (1995) y Saavedra (2003), que definen la resiliencia en 12 dimensiones (tabla 1 ):

\section{TABLA 1}

Factores de resiliencia Escala SV-RES

\begin{tabular}{|c|c|c|c|c|}
\hline \multirow{2}{*}{$\begin{array}{l}\text { Competencias } \\
\text { interaccionales } \\
\text { de Grotberg } \\
\text { (1995) }\end{array}$} & \multicolumn{4}{|c|}{$\begin{array}{l}\text { Niveles de estructuración de la conciencia de Saavedra } \\
\qquad(2003)\end{array}$} \\
\hline & $\begin{array}{l}\text { Condiciones } \\
\text { de base }\end{array}$ & $\begin{array}{l}\text { Visión de sí } \\
\text { mismo }\end{array}$ & $\begin{array}{l}\text { Visión del } \\
\text { problema }\end{array}$ & $\begin{array}{l}\text { Respuesta } \\
\text { resiliente }\end{array}$ \\
\hline $\begin{array}{l}\text { Yo soy, yo } \\
\text { estoy }\end{array}$ & $\begin{array}{l}\text { F1: } \\
\text { identidad }\end{array}$ & $\begin{array}{l}\text { F2: } \\
\text { Autonomía }\end{array}$ & $\begin{array}{l}\text { F3: } \\
\text { Satisfacción }\end{array}$ & $\begin{array}{l}\text { F4: } \\
\text { Pragmatismo }\end{array}$ \\
\hline Yo tengo & $\begin{array}{l}\text { F5: } \\
\text { Vinculos }\end{array}$ & F6: Redes & F7: Modelos & F8: Metas \\
\hline Yo puedo & $\begin{array}{l}\text { F9: } \\
\text { Afectividad }\end{array}$ & $\begin{array}{l}\text { F10: } \\
\text { Autoeficacia }\end{array}$ & $\begin{array}{l}\text { F11: } \\
\text { Aprendizaje }\end{array}$ & $\begin{array}{l}\text { F12: } \\
\text { Generatividad }\end{array}$ \\
\hline
\end{tabular}

Fuente: Villalta et al., 2007

La versión definitiva de la escala es de 60 ítems, con formato de respuesta Likert de 5 opciones que van de (1) Muy de Acuerdo a (5) Muy en Desacuerdo. Se han realizado estudios con altos indicadores validez y confiabilidad en diversas poblaciones de Chile: ciudades de Talca (Villalta et al., 2007), Curicó, Región Metropolitana (Villalta, 2010; Villalta \& Saavedra, 2012), y Concepción (Núñez, Parra, Villalta, \& Reyes, 2009); también ha probado alta validez en población de estudiantes migrantes en Barcelona (Sandín \& Sánchez, 2014), y un grupo de mujeres con cáncer de mama en Estado de México, México (Ocampo et al., 2011).

Estudios de la resiliencia con la escala SVRES en el campo educativo, ponen en evidencia que las dimensiones se comportan de modo diferente según el contexto social, la situación de adversidad, las características de los sujetos y el resultado positivo o adaptativo asociado.

Así, en la perspectiva de resistencia a situaciones estresantes, la investigación con estudiantes adolescentes chilenos de zonas urbanas, sugiere que los 12 componentes se pueden agrupar en dos: Confianza en uno mismo y confianza en los demás (Villalta et al., 2007); la comparación entre diversos grupos de edad pone en evidencia que los jóvenes entre 19 a 24 años puntúan más que los adultos entre 46 y 55 años en el puntaje general (Saavedra \& Villalta, 2008b); asimismo, el estudio en sujetos jóvenes y adultos drogodependientes participantes de una comunidad terapéutica reporta como fortalezas favorables a la recuperación las dimensiones: Redes, Aprendizajes e Identidad (Canelo, 2009); por su parte la dimensión Modelos está asociada al bienestar de los estudiantes en contextos sociales vulnerables (Villalta \& Saavedra, 2012).

Los estudios de resiliencia en perspectiva de relacionar situaciones de adversidad con rendimiento escolar a través de la escala SVRES, reportan que la relación entre resiliencia y logro educativo no es directa ni siempre evidente (Morales \& González, 2014; Villalta, 2010); no obstante, hay investigaciones en Chile con estudiantes en contexto social vulnerable y alto logro académico, donde destacan de modo significativo las dimensiones: Redes, Metas, Autoeficacia y Aprendizaje, en donde Metas es la dimensión más constante, incluso cuando se suma otros factores de riesgo tales como la separación de los padres y embarazo adolescente (Núñez et al., 2009; Villalta, 2010, 2013b). El análisis por género indica que el logro escolar de mujeres está asociado a las dimensiones Identidad y Vínculos (Villalta, 2013a).

Investigaciones de resiliencia con la escala SV-RES como elemento asociado al logro educativo en contextos sociales desfavorables, se han realizado también en estudiantes peruanos de sectores sociales vulnerables de Lima, comparando grupos de alto $y$ bajo rendimiento escolar, en donde se encuentra que las dimensiones: Identidad, Autonomía, Satisfacción, Pragmatismo, Vínculos, Autoeficacia, Aprendizaje y Generatividad son de puntuación más alta en el grupo de alto rendimiento académico (Delgado et al., 2011); estudios con inmigrantes latinoamericanos, rumanos, chinos y marroquís en Barcelona, reportan las dimensiones Identidad y Metas como aquellas que destacan en el grupo de alto rendimiento escolar (Sandín, Sánchez, 
Campo, \& Massot, 2014; Sandín \& Sánchez, 2013).

\section{Método}

Estudio cuantitativo de tipo analítico, descriptivo de respuestas a instrumento que mide resiliencia.

\section{Composición de la muestra}

Es una muestra no probabilística con dos criterios de inclusión: (1) Estudiantes de escuelas de sectores sociales vulnerables, definido por el nivel socioeconómico y los indicadores de vulnerabilidad social de las comunas de procedencia; y (2) rendimiento escolar, definido por las posiciones más alta y más baja en relación al promedio de su grupo nacional de referencia. La muestra final estuvo conformada por un total de 1909 adolescentes de sectores sociales vulnerables de la ciudad de Lima-Perú (764) y de Santiago de Chile (1 145), con edades que fluctúan entre los 13 y 18 años, 46\% hombres y $54 \%$ mujeres.

\section{Instrumento}

Se aplicó la Escala de Resiliencia SV-RES (Saavedra \& Villalta, 2008a) de 60 ítems, con alternativa de respuesta a cada ítem en un rango de 5 opciones: (1) Muy en desacuerdo, a (5) Muy de acuerdo. El puntaje máximo es de 300 puntos.

\section{Procedimiento de análisis de dato}

Para la validez se realizó el análisis factorial exploratorio (AFE); la confiabilidad se estudió con el alfa de Cronbach, y Análisis de Varianza Extraída (AVE). El AFE se realizó con el programa Factor 10.3 (Lorenzo-Seva \& Ferrando, 2006). El coeficiente alfa de Cronbach y los análisis descriptivos se calcularon con el SPSS 23.

\section{Resultados}

En primer lugar, se procedió a revisar el comportamiento de los 60 ítems que conformaban la prueba SV-RES en ambos grupos por separado - Lima y Santiago de Chile-, para una primera depuración de los ítems, con la que se eliminaron aquellos que no presentaron valores entre -1.5 y +1.5 . A raíz de ello, se eliminaron 30 ítems de manera que los mejores 30 fueron utilizados en los análisis psicométricos posteriores. Esto puso en evidencia que los 30 ítems que cumplían con esta primera condición de depuración eran diferentes por cada grupo nacional. La resiliencia está determinada por características intrínsecas de los grupos examinados, puestos en evidencia en las diferentes ponderaciones a los ítems de la escala.

Previo al análisis factorial exploratorio (AFE) con los 30 ítems del SVRES seleccionados, se efectuó el análisis de los datos mediante el estadístico de Mardia (1970), el cual, para el caso del grupo de Lima, indicó que había altas curtosis y asimetrías en las distribuciones de los ítems, y para el caso del grupo de Santiago de Chile, se encontró que había valores elevados en los mismos. Ante la falta de una distribución normal multivariada, se realizó el AFE mediante el procedimiento de mínimos cuadrados no ponderados (ULS) en ambas muestras por separado. Los resultados se presentan a continuación.

Análisis factorial exploratorio de la forma corta de la Escala de Resiliencia SV-RES en la muestra de Lima, Perú

Para el caso de la muestra de Lima, el análisis de la adecuación de la matriz indicó que se obtuvieron valores relevantes en el test de esfericidad de Bartlett (X2 (435) = 14610.2; p $<0.001)$, y un buen test de Kaiser-Meyer-Olkin (KMO = 0.976). Para la selección del número de factores que debían ser incluidos, se aplicaron los análisis paralelos de Horn (1965) y el método Hull (Lorenzo-Seva, Timmerman, \& Kiers, 2011), en ambos casos se debía retener 3 factores. Los 
análisis de la bondad de ajuste del modelo de 3 factores, indicó que se alcanzaron valores adecuados en el índice de la bondad de ajuste no normada de Tucker y Lewis $(\mathrm{NNFI}=0.962)$, un índice de la forma comparativa $(\mathrm{CFI}=0.986)$ y los índices de bondad de ajuste $(\mathrm{GFI}=1)$, y en el índice de bondad de ajuste ajustado (AGFI = 1). Adicionalmente, los índices de la simplicidad de la solución factorial alcanzaron adecuados valores en el índice de simplicidad de Bentler (S $=0.987$ ), un índice de simplicidad de la carga factorial ( $\mathrm{LS}=0.48$ ), y el análisis de los residuales indicó que el residuo cuadrático medio presentó un valor de $($ RMSR $)=0.026$, el cual de acuerdo al criterio de Kelley $(\mathrm{RMSR}=0.0362)$, presentó un modelo aceptable.

El análisis de la estructura factorial (tabla 2) indicó que el primer factor explica el $49.47 \%$ de la varianza total; el segundo factor, el 5.36\%; y el tercer factor, el 3.1\%, lo que evidencia que el modelo permitió explicar el $57.93 \%$ dela varianza total.

Finalmente, la revisión de la congruencia de la solución del AFE, indicó que los índices de la simplicidad de la solución factorial arrojan valores óptimos en el índice de simplicidad de Bentler $(S=0.987)$ y el índice de simplicidad de la carga factorial $(\mathrm{LS}=0.48)$.

\section{TABLA 2}

Análisis factorial exploratorio de la muestra de alumnos de la ciudad de Lima

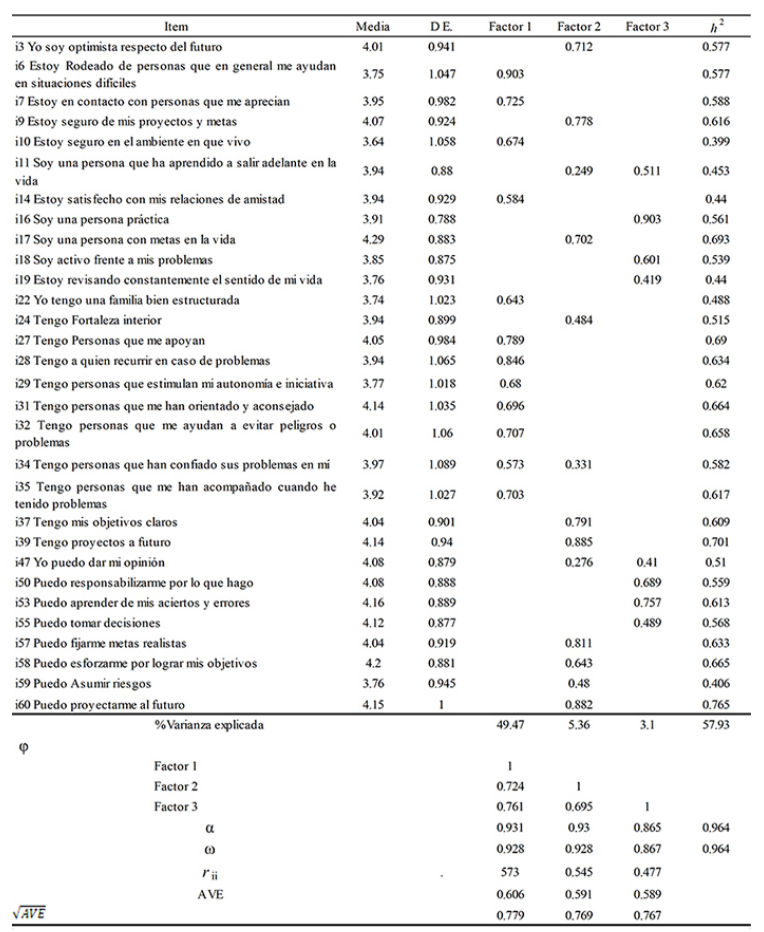

Nota.r $r_{i 1}:$ correlación inter-ítem promedio. AVE: varianza extraída promedio. a y w: coeficientes de confiabilidad. j: correlación interfactorial. * coeficiente corregido por errores correlacionados. Fuente: elaboración propia.

Evidencias de validez de la forma corta de la Escala de Resiliencia SV-RES, basada en la estructura interna en la muestra de Lima

La validez convergente corresponde al promedio de varianza extraída (AVE), el mismo que debería ser superior a 0.5 de acuerdo con Fornell y Larcker (1981). La validez discriminante se evaluó mediante la comparación de la raíz cuadrada de AVE con la correlación interfactorial de los otros constructos $(\phi)$, de manera que se espera que el valor de sea mayor que $\phi$ (correlación interfactorial). En la tabla 2, se aprecia que tanto los valores de AVE, como los valores de las raíces cuadradas de AVE superaron los criterios planteados de manera que los ítems conforman escalas que son consistentes y a la vez diferentes. 
Los tres factores emergentes de los análisis realizados a los ítems de la Escala SV-RES se definen a continuación:

Factor 1: compromiso con el entorno cercano

Agrupa ítems de la escala SV-RES que refieren a la percepción de vínculos fecundos con el entorno social cercano -familia y amigos-, con los cuales el adolescente se siente seguro, satisfecho y comprometido.

Factor 2: visión optimista del futuro

Agrupa ítems de la escala SV-RES que guardan en común el sentido positivo que se otorga al futuro, en relación al cual se siente capaz de trazar objetivos y desarrollar estrategias para alcanzarlo.

Factor 3. confianza en las propias capacidades de acción

Agrupa ítems que guardan en común la seguridad en las decisiones propias, sustentada en la confianza en las propias capacidades para salir adelante en la vida, aprender de aciertos y errores, tomar decisiones y revisar constantemente el sentido de la vida.

\section{Análisis de la confiabilidad de la forma corta de la Escala de Resiliencia SV-RES en la muestra de Lima}

Para analizar las evidencias de confiabilidad, se calculó el coeficiente alfa de Cronbach (Tavakol \& Dennick, 2011). Como se utilizó el análisis factorial exploratorio, se calculó el coeficiente omega de Mac Donald (Gignac, 2009). Los resultados indicaron que se alcanzaron elevados coeficientes alfa de Cronbach, los cuales fluctuaron entre 0.865 y 0.964 . Adicionalmente, los coeficientes omega presentaron valores similares, que oscilaron entre 0.865 y 0.964 .

\section{Análisis factorial exploratorio de la forma corta de la Escala de Resiliencia SV-RES en la muestra de Santiago de Chile}

En el caso de la muestra de Santiago de Chile, el análisis de la adecuación de la matriz indicó valores relevantes en el test de esfericidad de Bartlett $(\mathrm{X} 2(435)=11833.3 ; \mathrm{p}<0.001)$, y un elevado valor en el test de Kaiser-MeyerOlkin $(\mathrm{KMO}=0.951)$. El número de factores fue determinado por los análisis paralelos de Horn y de Hull, cuyos resultados sugieren que se debían retener 4 factores. Los análisis estadísticos del modelo factorial de 4 ítems, indican adecuados valores en el Índice de la bondad de ajuste no normada de Tucker y Lewis $(\mathrm{NNFI}=0.929)$, en el Índice de la forma comparativa (CFI = 0.957) y los índices de Bondad de ajuste (GFI = 0.995), así como en el índice de bondad de ajuste ajustado $(\mathrm{AGFI}=0.996)$. Adicionalmente los índices de la simplicidad de la solución factorial, indicaron que se alcanzaron adecuados valores en el Índice de Simplicidad de Bentler $(S=0.968)$, y un índice de Simplicidad de la carga factorial $(\mathrm{LS}=0.365)$. El análisis del residuo cuadrático medio alcanzó un valor de (RMSR) $=0.086$, el cual, de acuerdo al criterio de Kelley (RMSR = 0.076), es aceptable.

El análisis de la estructura factorial (tabla 3) indicó que el primer factor explica el $49.47 \%$ de la varianza total el segundo factor, el 5.36\%; y el tercer factor, el 3.1\%, lo que evidencia que el modelo permitió explicar el $57.93 \%$ de la varianza total. Finalmente, los índices que miden la congruencia de la solución del AFE permitieron apreciar valores adecuados en el Índice de simplicidad de Bentler $(S=0.905)$, un índice de simplicidad de la carga factorial (LS = 0.403), y el análisis de los residuos cuadráticos (RMSR) alcanzó un valor de 0.0286, que es adecuado en comparación con el criterio de Kelley $($ RMSR $=0.0296)$. 
TABLA 3

Análisis factorial exploratorio de la muestra de alumnos de la ciudad de Santiago de Chile

\begin{tabular}{|c|c|c|c|c|c|c|c|}
\hline item & Media & DE & Factor 1 & Factor 2 & Factor 3 & Factor 4 & $h^{2}$ \\
\hline i2 Yo soy una persona con buena autoestima & 4.04 & 0.982 & 0.632 & & & & 0.398 \\
\hline $\begin{array}{l}\text { i6 Estoy rodeado de personas que en general me } \\
\text { ayudan en situaciones difficiles }\end{array}$ & 4.18 & 0.955 & & & 0.476 & & 0.387 \\
\hline ig Estoy seguro de mis proyectos y metas & 4.26 & 0.863 & & 0.394 & & & 0.485 \\
\hline il Estoy seguro en el ambiente en que vivo & 3.97 & 1.08 & 0.569 & & & & 0.336 \\
\hline il2 Soy un modelo positivo para otras personas & 3.59 & 0.975 & 0.509 & & & & 0.377 \\
\hline $\begin{array}{l}\text { lil Estoy bien integrado en mi lugar de trabajo o } \\
\text { estudio }\end{array}$ & 4.06 & 0.879 & 0.551 & & & & 0.37 \\
\hline il5 Estoy satisfecho con mis relaciones afectivas & 4.02 & 1.001 & 0.339 & & & & 0.414 \\
\hline il8 Soy activo frente a mis problemss & 4.19 & 0.917 & & & & 0.462 & 0.408 \\
\hline $\begin{array}{l}\text { il9 Estoy revisando constantemente el sentido de } \\
\text { mivida }\end{array}$ & 4.04 & 0.934 & & & & 0.449 & 0.394 \\
\hline i20 Estoy generando soluciones a mis problemas & 4.2 & 0.865 & & & . & 0.557 & 0.479 \\
\hline i2l Yo tengo relaciones personales confiables & 4.15 & 0.899 & 0.736 & & & & 0.534 \\
\hline i22 Tengo una fanilia bien estructurada & 4.14 & 1.064 & 0.609 & & & & 0.479 \\
\hline 123 Tengo relaciones afectivas sólidas & 3.94 & 0.981 & 0.653 & & & & 0.6 \\
\hline i2s Tengo una vida con sentido & 4.23 & 0.907 & 0.745 & & & & 0.561 \\
\hline I26 Tengo acceso a servicios Sociales-públicos & 3.77 & 1.062 & & 0.564 & & & 0.322 \\
\hline $\begin{array}{l}\text { i29 Tengo personas que estimulan mi autonomia e } \\
\text { iniciativa }\end{array}$ & 4.19 & 0.856 & 0.317 & & & & 0.403 \\
\hline $\begin{array}{l}\text { ios Tengo satisfacción con lo que he logrado en la } \\
\text { vida }\end{array}$ & 4.05 & 0.967 & 0.468 & & & & 0.413 \\
\hline 137 Tengo mis objetivos claros & 4.23 & 0.908 & 0.314 & 0.449 & & & 0.467 \\
\hline $\begin{array}{l}\text { i3s Tengo personas con quien enfrentar los } \\
\text { problemes }\end{array}$ & 4.28 & 0.887 & & & 0.345 & & 0.429 \\
\hline i 40 Tengo problemss que puedo solucionar & 4.2 & 0.89 & & 0.371 & & & 0.422 \\
\hline iAl Yo puedo hablar de mis emociones & 3.8 & 1.111 & & & 0.723 & & 0.544 \\
\hline i42 Puedo expresar afecto & 4.22 & 0.909 & & & 0.577 & & 0.448 \\
\hline j43 Puedo confiar en las personas & 3.92 & 1.034 & & & 0.637 & & 0.47 \\
\hline 146 Puedo nsolver problemas de manera efectiva & 4.09 & 0.899 & & 0.487 & & & 0.441 \\
\hline i47 Puedo dar mi opinión & 4.33 & 0.829 & & 0.567 & & & 0.454 \\
\hline 448 Puedo buscar ayuda cuando la neeesito & 4.19 & 0.972 & & & 0.628 & & 0.5 \\
\hline is Puedo ser creativo & 4.26 & 0.876 & & 0.613 & & & 0.395 \\
\hline $\begin{array}{l}\text { is Puedo colaborar con otros para mejorar la vida } \\
\text { en la comunidad }\end{array}$ & 4.07 & 0.9 & & 0.517 & & & 0.411 \\
\hline $\begin{array}{l}\text { is Puedo generar estrategias para solucionar mis } \\
\text { problemas }\end{array}$ & 4.24 & 0.854 & & 0.572 & & & 0.468 \\
\hline is9 Puedo asumir riesgos & 4.31 & 0.796 & & 0.728 & & & 0.475 \\
\hline \% Varianza explicada & & & 31.73 & 7.17 & 6.88 & 5.45 & 51.23 \\
\hline & & & 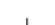 & & & & \\
\hline $\begin{array}{l}\text { Fatoror } 1 \\
\text { Fatcor } 2\end{array}$ & & & & 1 & & & \\
\hline $\begin{array}{l}\text { Factor } 2 \\
\text { Factor } 3\end{array}$ & & & 0.527 & 0.631 & 1 & & \\
\hline Factor 4 & & & 0.581 & 0.657 & 0.692 & 1 & \\
\hline$\alpha$ & & & 0.918 & 0.824 & 0.82 & 0.765 & 0.919 \\
\hline (1) & & & 0.823 & 0.799 & 0.798 & 0.765 & 0.925 \\
\hline$r_{\text {ii }}$ & & & 0.301 & 0.32 & 0.352 & 0.431 & \\
\hline AVE & & & 0.51 & 0.502 & 0.522 & 0.514 & \\
\hline$\sqrt{A V E}$ & & & 0.714 & 0.709 & 0.722 & 0.717 & \\
\hline
\end{tabular}

Nota.r $r_{i i}:$ correlación inter-ítem promedio. AVE: varianza extraída promedio. a y w: coeficientes de confiabilidad. j: correlación interfactorial. * coeficiente corregido por errores correlacionados. Fuente: Elaboración propia.

Evidencias de validez de la forma corta de la Escala de Resiliencia SV-RES, basada en la estructura interna en la muestra de Santiago de Chile

Con procedimientos y criterios estadísticos similares a los empleados para el análisis de validez convergente en la muestra de Lima, Perú, en la tabla 5 se aprecia que tanto los valores de AVE como los valores de las raíces cuadradas de AVE superaron los criterios planteados (AVE $>0.5)$, de tal forma que los ítems constituyen factores que son consistentes para medir aspectos específicos del constructo, y que a la vez son diferentes entre sí.

Los cuatro factores emergentes de los análisis realizados a los ítems de la Escala SV-RES se definen a continuación:
Factor 1: visión positiva de sí mismo

Agrupa ítems que tienen en común los juicios positivos que los adolescentes tienen sobre sí mismos, lo logrado en sus vidas y las relaciones afectivas que establecen con las personas que los rodean.

Factor 2: predisposición a la acción

Agrupa ítems que tienen en común la seguridad y confianza para trazarse objetivos, resolver problemas, opinar, crear, colaborar con otros, asumir riesgos.

Factor 3: relación positiva con el entorno

Agrupa ítems que tienen en común el vínculo positivo que el adolescente establece con la gente del entorno para expresar afectos, sentirse apoyado para enfrentar problemas, hablar de sus emociones y buscar ayuda.

Factor 4: pragmatismo

Agrupa los ítems que tienen en común la orientación a la acción de los adolescentes para revisar constantemente el sentido de su vida y actuar para generar soluciones a situaciones específicas.

\section{Análisis de la confiabilidad de la forma corta de la Escala de Resiliencia SV-RES en la muestra Santiago de Chile}

EL análisis de la confiabilidad de las puntuaciones se realizó con los coeficientes alfa de Cronbach y Omega de Mac Donald, los hallazgos permitieron observar que se alcanzó coeficientes elevados, de manera que los coeficientes alfa de Cronbach fluctuaron entre 0.765 y 0.919 , mientras que los coeficientes omega presentaron valores similares, con valores que oscilaron entre 0.765 y 0.925 . 


\section{Discusión y conclusiones}

La resiliencia puede ser considerada una capacidad humana universal (Grotberg, 1995), pero según lo demuestran el análisis de comportamiento de los datos en el presente estudio, se desarrolla de modo específico para situaciones específicas. Los factores personales y externos son relevantes en la resiliencia del individuo (Cabanyes, 2010), y la interacción de dichos factores dificulta definir criterios de medición únicos en contextos aparentemente similares. Los aspectos culturales y de socialización definen procesos de resiliencia vinculados al rendimiento escolar, diferentes entre poblaciones de adolescentes de Lima y de Santiago de Chile.

En consecuencia, la medición de la resiliencia vinculada al rendimiento escolar en adolescentes funciona mejor de modo independiente entre las poblaciones de Lima y Santiago de Chile. Las versiones cortas de 30 ítems, extraídas del análisis de datos de la Escala de Resiliencia SVRES, refieren a factores diferentes respecto a su cantidad - tres para la muestra de Lima y 4 para la muestra de Santiago de Chile-y a su definición. Insistir en una versión común, aunque deseable para avanzar en criterios generales de medición, perjudicaría la precisión descriptiva de la resiliencia para ambas poblaciones, haciendo invisibles factores emergentes que son propios y relevantes para cada población.

Aunque ambas escalas cortas miden la resiliencia asociada al logro escolar, para el caso de la muestra de Lima esta destaca en sus tres factores - Compromiso con el entorno cercano, visión optimista del futuro y confianza en las propias capacidades de acción - la importancia del entorno social cercano y el compromiso con el futuro; en tanto que los cuatro factores de la muestra de Santiago de Chile - Visión positiva de sí mismo, predisposición a la acción, relación positiva con el entorno y pragmatismo- ponen el acento en un locus de control más interno y centrado en la acción sobre el presente.

Ambas escalas confluyen de modo especial en el valor de la acción resiliente para afrontar situaciones problemáticas, y conservan la virtud de ser sensibles a las características culturales de cada grupo. Ambas escalas redefinen los factores o dimensiones de la resiliencia de la Escala SV-RES para precisar la asociación con el rendimiento escolar según cada contexto nacional de vulnerabilidad social.

\section{Agradecimientos}

Auspiciado por la Dirección de Investigación Científica y Tecnológica (DICYT) de la Universidad de Santiago de Chile.

\section{Referencias}

Brandão, J. M., Mahfoud, M., \& GianordoliNascimento, I. F. (2011). The construction of the concept of resilience in psychology: Discussing the origins of resilience. Paideia, 21 (49), 263-271. https://doi.org/10.1590/S 0103-863X2011000200014

Cabanyes, J. (2010). Resilience: An approach to the concept. Revista de Psiquiatria y Salud Mental, 3(4), 145-151. https://doi.org/10.1 016/j.rpsm.2010.09.003

Canelo, P. (2009). Factores resilientes en usuarios Programa de Atención para Drogadictos" Caleta Sur". (Licenciada en Psicología), Universidad Academia de Humanismo Cristiano. Santiago.

Cortés, B. G. M., \& Cruz, L. P. (2011). Resilience: Is it possible to measure and influence it? Salud Mental, 34(3), 237-246.

Delgado, A., Escurra, L., Villalta, M., Saavedra, E., Atalaya, M., Pequeña, J., ... \& Rodríguez, R. (2011). Resiliencia en Alumnos con Alto y Bajo Rendimiento de Quinto Año de secundaria de Instituciones Educativas Estatales de Zonas UrbanoMarginales de la Ciudad de Lima. Ciencia y Aprendizaje, 3(1), 45-70.

Fergus, S., \& Zimmerman, M. A. (2005). Adolescent resilience: a framework for understanding healthy development in the face of risk. Annu Rev Public Health, 26, 399-419. https://doi.org/10.1146/annurev. publhealth.26.021304.144357 
Fornell, C., \& Larcker, D. F. (1981). Evaluating Structural Equation Models with Unobservable Variables and Measurement Error. Journal of Marketing Research, 18(1), 39-50. https://doi.org/10.2307/3151312

Gaxiola, J., González, S., \& Gaxiola, E. (2013). Self-regulation, resilience and educational goals: Variables protecting the academic performance of high school students. Revista Colombiana de Psicologia, 22(2), 241-252.

Gaxiola, J. C., Frías, M., Hurtado, M. F., Salcido, L. C., \& Figueroa, M. (2011). Validación del inventario (IRES) en una muestra del noroeste de México. Enseñanza e Investigación en Psicología, 16(1), 73-83.

Gibbs, S., \& Miller, A. (2014). Teachers resilience and well-being: A role for educational psychology. Teachers and Teaching: Theory and Practice, 20(5), 609-621. https://doi.org $/ 10.1080 / 13540602.2013 .844408$

Gignac, G. E. (2009). Psychometrics and the measurement of emotional intelligence. In C. Stough (Ed.), Assessing Emotional Intelligence (pp. 9-40): Springer.

Gloria, C. T., Faulk, K. E., \& Steinhardt, M. A. (2013). Positive affectivity predicts successful and unsuccessful adaptation to stress. Motivation and Emotion, 37(1), 185-193. https://doi.org/10.1007/s11031-0 $12-9291-8$

Grotberg, E. (2005). Nuevas tendencias en resiliencia Resiliencia. Descubriendo las propias fortalezas (pp. 19-30). Buenos Aires: Paidós.

Grotberg, E. (1995). A guide to promoting resilience in children: strengthening the human spirit. The Hague, The Netherlands: Bernard Van Leer Foundation.

Horn, J. L. (1965). A rationale and test for the number of factors in factor analysis. Psychometrika, 30(2), 179-185.

Kotzé, M., \& Kleynhans, R. (2013). Psychological resource capacities (hope, optimism and resilience) as predictors of burnout in first-year students at a higher education institution. International Journal of Learning in Higher Education, 19(2), 31-50.
Lever, J. P., \& Valdez, N. E. G. (2010). Construction of a measurement scale of resilience in Mexicans (RESI-M). Interdisciplinaria, 27(1), 7-22.

Lorenzo-Seva, U., \& Ferrando, P. J. (2006). FACTOR: A computer program to fit the exploratory factor analysis model. Behavior research methods, 38(1), 88-91. https://doi.o $\mathrm{rg} / 10.3758 / \mathrm{BF} 03192753$

Lorenzo-Seva, U., Timmerman, M. E., \& Kiers, H. A. (2011). The Hull method for selecting the number of common factors. Multivariate Behavioral Research, 46(2), 340-364.

Luthar, S. S., Cicchetti, D., \& Becker, B. (2000). The construct of resilience: A critical evaluation and guidelines for future work. Child Development, 71(3), 543-562.

Llobet, V., \& Wegsman, S. (2004). Enfoque de resiliencia en los proyectos sociales. Perspectivas y desafíos. Revista de Psicología, 13(1), 143-152.

Mardia, K. V. (1970). Measures of multivariate skewness and kurtosis with applications. Biometrika, 57(3), 519-530.

Morales, M., \& González, A. (2014). ResilienciaAutoestima-Bienestar psicológico y Capacidad intelectual de estudiantes de cuarto medio de buen rendimiento de liceos vulnerables. Estudios Pedagógicos, 40(1), 215-228.

Morelato, G. S. (2014). Assessment of resilience factors in argentines children in familiar vulnerability conditions. Universitas Psychologica, 13(4), 1473-1488. https://doi. org/10.11144/Javeriana.UPSY13-4.efrn

Moreno, R., \& Saiz, C. (2014). Factores resilientes en los futuros maestros. International Journal of Developmental and Educational Psychology, 1(3), 475-488.

Núñez, M., Parra, M., Villalta, M., \& Reyes, F. (2009). Factores resilientes en adolescentes de contexto de alta vulnerabilidad escolar en la ciudad de Concepción, Chile. Ciencia y Aprendizaje, 1(1), 41-63.

Ocampo, J., Valdéz, J., González-Arratia , N., Andrade- Palos, P., Oblitas-Guadalupe, L., \& García-Fabela, R. (2011). Variables 
Psicológicas Predictoras de la Calidad de Vida en Pacientes con Cáncer de Mama. Clínica Psicológica - Fundación Aiglé XX(3), 265-269.

Ospina, D. E. (2007). La medición de la resiliencia. Investigación y Educación en Enfermería, 25(1), 58-65.

Peralta, S. C., Ramírez, A. F., \& Castaño, H. (2006). Factores resilientes asociados al rendimiento académico en estudiantes pertenecientes a la Universidad de Sucre (Colombia). Psicología desde el Caribe(17), 196-2019.

Saavedra, E., \& Villalta, M. (2008a). Escala de resiliencia SV-RES, para jóvenes y adultos. Santiago: Ceanim.

Saavedra, E., \& Villalta, M. (2008b). Medición de la características resilientes, un estudio comparativo en personas entre 15 y 65 años. LIBERABIT, 14, 31-40.

Saavedra, E. (2003). El enfoque Cognitivo Procesal Sistémico, como posibilidad de intervenir educativamente en la formación de sujetos Resilientes: estudio de casos. Tesis Doctoral, Universidad de Valladolid, España.

Salgado, A. C. (2005). Métodos e instrumentos para medir la resiliencia: una alternativa peruana. LIBERABIT, 11, 41-48.

Sandín, M., \& Sánchez, A. (2014). Beyond Compulsory Schooling: Resilience and Academic Success of Immigrant Youth. Procedia-Social and Behavioral Sciences, 132, 19-24.

Sandín, M., Sánchez, A., Campo, J. d., \& Massot, M. (2014). Resiliència i èxit educatiu en l'alumnat de secundària de procedència estrangera. In Generalitat de Catalunya (Ed.), Recerca i immigració VI. Convocatòria d'ajuts 2010 (Vol. 10, pp. 119-141). Catalunya.

Sandín, M. P., \& Sánchez, A. (2013). Resiliencia y persistencia académica en estudiantes inmigrantes. Revista Iberoamericana de Educación (OEI), 62 (1), 1-11.

Tavakol, M., \& Dennick, R. (2011). Making sense of Cronbach's alpha. International journal of medical education, 2, 53-55.
Villalta, M. (2010). Factores de resiliencia asociados al rendimiento académico en estudiantes de contextos de alta vulnerabilidad social. Revista de Pedagogia, 31(1), 159-190.

Villalta, M. (2013a). Resiliencia y rendimiento escolar a través de la escala SV-RES. In J. C. Gaxiola \& J. Palomar (Eds.), Estudios de resiliencia en América Latina (Vol. 2, pp. 37-51). México D.F.: Pearson Educación, Universidad Iberoamericana.

Villalta, M. (2013b). Resiliencia y rendimiento escolar en contextos de alta vulnerabilidad social de chile. Uso de la escala SV-RES. In J. Madariaga, A. Forés, E. Goñi, M. d. 1. O. Palma, O. Pérez, A. Rodriguez, C. Villalba, \& G. Gil (Eds.), La construcción social de la resiliencia (pp. 143-149). Bilbao, España: Universidad del Pais Vasco.

Villalta, M., \& Delgado, A. (2013). Propuesta de Escala de Resiliencia para adolescentes chilenos: SV-RES Abreviada. In J. M. Madariaga, A. Forés, E. Goñi, M. d. 1. O. Palma, O. Pérez-Muga, A. Rodriguez, C. Villalba, M., \& G. Gil (Eds.), La construcción social de la Resiliencia. (pp. 21-29). Bilbao: Servicio Editorial de la Universidad del País Vasco.

Villalta, M., \& Saavedra, E. (2012). Cultura escolar, prácticas de enseñanza y resiliencia en alumnos y profesores de contextos sociales vulnerables. Universitas Psychologica, 11(1), 67-78.

Villalta, M., Saavedra, E., \& Escurra, L. (2007). Factores de resiliencia en estudiantes secundarios. Una propuesta de medición. Paper presented at the VII Jornadas Internacionales de Psicología Educacional, Pucón, Chile.

\section{Notas}

* Artículo de investigación. 